\title{
Equations of Spheroid and Ellipsoid Surface Area by Analytical Method
}

\author{
Abolfazl Soltani * \\ Department of Physics, University of Birjand, Birjand, Iran \\ Email: soltani.a.physics@gmail.com
}

\begin{abstract}
We do not have any exact equation for ellipsoid and subsequently spheroid surface area. Here we will obtain the equations of Spheroid and Ellipsoid surface area by analytical method.
\end{abstract}

Keywords: Oblate spheroid, Ellipsoid, Prolate spheroid, Surface area

\section{Oblate Spheroid Surface Area}

As you know, an oblate spheroid with scales $a=a_{0}>\left(c_{0}=b_{0}\right)$ is obtained from the whole rotation of an ellipse with semi-major axis $a_{0}$ and semi minor axis $b_{0}$ around the $a_{0}$ axis. Now we want to calculate the surface area of this oblate spheroid by the method which we described in the previous preprint [1]. Consider Figure 1. In this figure, an oblate spheroid will draw from the complete rotation of the blue ellipse with scales $a_{0}$ and $b_{0}$ around the $x$-axis.

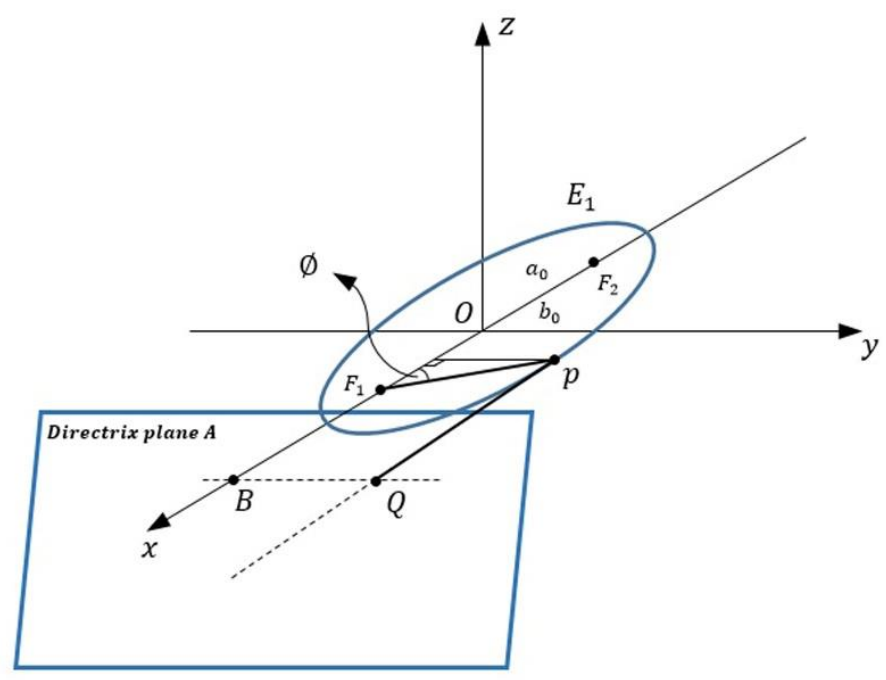

Fig. 1. As shown in the figure, the blue ellipse is on the horizontal plane $x y$. From the rotation of the blue ellipse around the $x$-axis, an oblate spheroid will build. The plane $A$ is located at a vertical distance $\frac{a}{e}$ from the origin $O$ and is perpendicular to the $x$ axis and we call it the ellipsoid Directrix plane.

In the ellipse in figure 1 we have [2][3]:

$$
\frac{F_{1} P}{Q P}=\frac{r}{d+r \operatorname{Sin} \theta \operatorname{Cos} \emptyset}=e_{0}
$$

In this equation, $Q P$ is the vertical distance from point $P$ to the ellipsoid directrix plane, ie plane $A$. This plane is perpendicular to the $x$-axis and its vertical distance to point $O$ is equal to $a_{0} / e_{0} . d$ is the distance between $F_{1}$ and $B$ (point $B$ is on the plane $A$ ) and we have: $d=\frac{a_{0}}{e_{0}}-e_{0} a_{0}$. Equation 1 stands for all the points $P$ on the surface of the oblate 
spheroid, due to we can build a oblate spheroid by rotation of the ellipse in Fig. 1 around $x$-axis. In equation $1, r \sin \theta \cos \varnothing$ is the shadow of $F_{1} P$ line on the $x$ axis and is written in spherical polar coordinates $(r . \theta . \varnothing)$ based on figure 2 , because the spheroid is a threedimensional object. $d=\frac{a_{0}}{e_{0}}-e_{0} a_{0}$ and therefore, from 1 we have:

$$
r-r e_{0} \operatorname{Sin} \theta \operatorname{Cos} \emptyset=a_{0}\left(1-e_{0}^{2}\right)
$$

like the previous article [1], we consider the left side of the equation 2 equal to $\eta$. In such a case, equation 2 is the equation of a sphere with radius $a_{0}\left(1-e_{0}^{2}\right)$; Because of $r^{2}\left(\operatorname{Sin}^{2} \alpha+\operatorname{Sin}^{2} \beta+\operatorname{Sin}^{2} \gamma\right)=a^{2}$ or $r=a$ is the equation of a sphere.

$$
\eta=a_{0}\left(1-e_{0}^{2}\right)
$$

And so the surface area of our oblate spheroid is equal to the area of its equivalent sphere.

$$
A_{\text {oblate spheroid }}=A_{\text {equivalent sphere }}=4 \pi \eta^{2}=4 \pi a_{0}^{2}\left(1-e_{0}^{2}\right)^{2}=4 \pi a_{0}^{2}-8 \pi a_{0}^{2} e_{0}^{2}+4 \pi a_{0}^{2} e_{0}^{4}
$$

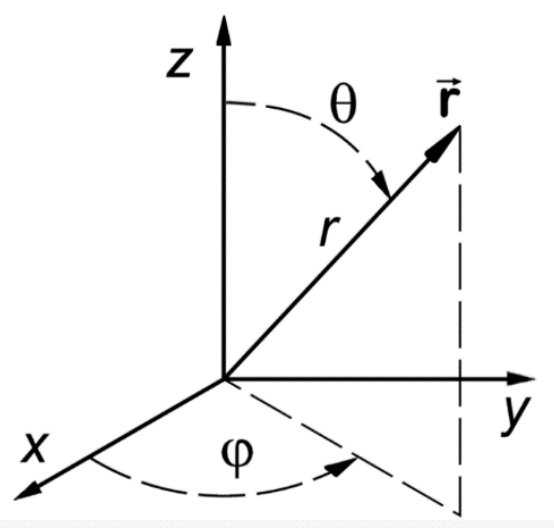

Fig .2.

\section{Ellipsoid Surface Area}

In an oblate spheroid we have:

$$
b_{0}=c_{0}=a_{0} \sqrt{\left(1-e_{0}^{2}\right)} \Rightarrow\left(1-e_{0}^{2}\right)=\frac{b_{0}^{2}}{a_{0}^{2}} \Rightarrow\left(1-e_{0}^{2}\right)^{2}=\frac{b_{0}^{4}}{a_{0}^{4}}
$$

Substituting in equation 4 :

$$
A_{\text {oblate spheroid }}=4 \pi a_{0}^{2}\left(1-e_{0}^{2}\right)^{2}=4 \pi \frac{b_{0}^{4}}{a_{0}^{2}}
$$

Equations 4 and 5 are only the surface area of oblate spheroid. Now the question is how to obtain the equations of surface area of prolate spheroid and ellipsoid? Well, there is no problem with that. It is important to note that the equation of the area of an oblate spheroid, equation 5, is the equation of the area of an ellipsoid in a particular state (boundary conditions), which we have written it in a special way. The general equation of the surface area of an ellipsoid, with scales $a_{0} \neq b_{0} \neq c_{0}$, should be a function of the $a_{0}, b_{0}, c_{0}$. According to Figure 3 , it seems that $b_{0}$ and $c_{0}$ must have an equal share in the ellipsoid surface area equation. Therefore, based on equation 5, we write the equation for an ellipsoid as follows: 


$$
A_{\text {Ellipsoid }}=4 \pi \frac{b_{0}^{2} c_{0}^{2}}{a_{0}^{2}}
$$

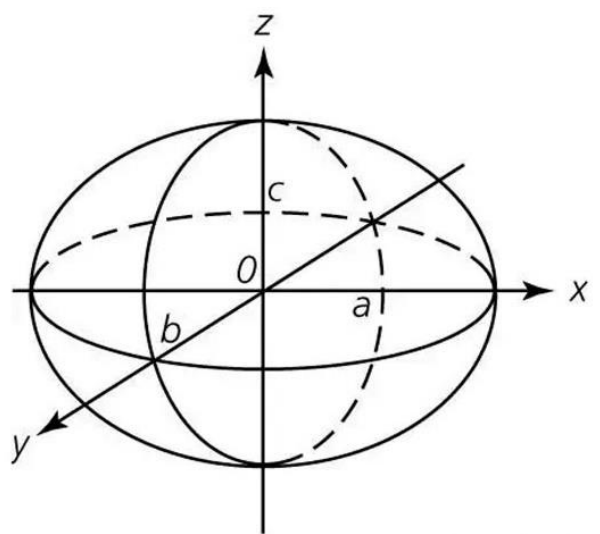

Fig. 3. An ellipsoid

For a Sphere we have: $a_{0}=b_{0}=c_{0}=r_{0}$ So based on 6 we have: $A_{\text {Sphere }}=4 \pi r_{0}^{2}$.

In a prolate spheroid we have $b_{0}>\left(a_{0}=c_{0}\right)$; So $A_{\text {Prolate spheroid }}=4 \pi \frac{a_{0}^{4}}{b_{0}^{2}}$ which is unequal to $A_{\text {Oblate spheroid }}=4 \pi \frac{b_{0}^{4}}{a_{0}^{2}}$.

\section{Discussion}

In this article we use from the concept of Directrix Plane of an ellipsoid to obtain the equation of surface area of an ellipsoid. This concept seems to be entering Geometry and Mathematics for the first time.

\section{References:}

[1]. Soltani, A. Exact Calculation of Ellipse Perimeter by Analytical Method, Cambridge Open Engage, DOI: https://doi.org/10.33774/coe-2022-rskdp

[2]. Silverman, R. Calculus with Analytic Geometry (Prentice-Hall, Inc. New Jersey. 1985), pp. 628-641

[3]. Johnson, R. Calculus with Analytic Geometry (Allyn and Bacon. Inc. Boston, ed. 4), pp. 536-539 